\title{
MICROSTRUCTURE EVOLUTION DURING NORMAL GRAIN GROWTH UNDER HIGH PRESSURE IN 2-D ALUMINIUM FOILS
}

\author{
V. SURSAEVA ${ }^{\mathrm{a}, *}$, S. PROTASOVA ${ }^{\mathrm{a}}$, W. LOJKOWSKI ${ }^{\mathrm{b}}$ \\ and J. JUN ${ }^{\mathrm{b}}$ \\ ${ }^{a}$ Institute of Solid State Physics, Russian Academy of Sciences,
} Chernogolovka. 142432 Russia; ${ }^{\text {b }}$ UNIPRESS, High Pressure Research Centre, Polish Academy of Sciences, 01-142 Warsaw, Sokolowska 29, Poland

(Received in final form 28 September 1997)

We investigated the effect of high hydrostatic pressure on the normal grain growth in 2-D aluminium foils. The time dependence of the mean grain area was obtained. It was shown that normal grain growth takes place both at atmospheric pressure and under high hydrostatic pressure. The grain growth rate decreases by a factor 1.3 under high pressure. The activation volume for grain growth was 0.13 of the atomic volume. It was shown that high pressure strongly influences the ratio of low angle boundaries and general boundaries at the early stages of secondary recrystallization.

Keywords: Normal grain growth; 2-D grain growth; Low-angle boundary distribution; High hydrostatic pressure

\section{INTRODUCTION}

The investigation of the hydrostatic pressure effect on the kinetics of a thermally activated process permits to assess the activation volume $V^{*}$. The activation volume enters as follows in the equation for the activation free energy $G^{*}$ :

$$
G^{*}=Q^{*}-T S^{*}+p V^{*},
$$

\footnotetext{
* Corresponding author.
} 
where $Q^{*}, S^{*}$ are the energy and entropy of activation, respectively, $T$ is the temperature and $p$ is the pressure, $V^{*}$ is the volume expansion of the crystal related to atomic rearrangements during the thermally activated process.

Knowledge of $V^{*}$ permits therefore to draw conclusions concerning the mechanism of the process investigated. $V^{*}$ can be obtained from the equation

$$
V^{*}=-R T \ln (v) / p
$$

where $R$ is the gas constant, and $v$ is the rate of the process investigated.

There are a number of papers where the influence of high pressure on diffusion was studied (Curtin et al., 1965; Buescher et al., 1973; Kedves and Erdelyi, 1989; Kohler et al., 1992; Mehrer, 1996). It was shown that the diffusion processes are suppressed by pressure and $V^{*}$ varies in range from 0 to $0.9 \Omega$ ( $\Omega$ is the atomic volume). $V^{*}$ values close to $0 \Omega$ may indicate an interstitial diffusion mechanism whereas $V^{*}$ values close to $1 \Omega$ are usually attributed to a vacancy diffusion mechanism.

Much less is known about the pressure effect on grain boundary (GB) diffusion (Martin et al., 1967; Erdelyi et al., 1987; Vieregge et al., 1991; Lojkowski et al., 1995; Lojkowski, 1996). Activation volumes in the range $0-1 \Omega$ have been found indicating that both interstitial and vacancy $G B$ diffusion mechanisms are active. The question arises as to what information about the GB migration mechanism can be drawn from high pressure studies. There are only a few papers where influence of high pressure on the grain growth (Hahn and Gleiter, 1979; Lojkowski et al., 1995) and migration of individual grain boundaries (Molodov et al., 1984; 1994) was studied. It was found, that the activation energy and activation volume for grain boundary migration in aluminium bicrystals are larger than that for grain growth in aluminium polycrystal (Lojkowski et al., 1995; Molodov et al., 1984; 1994) and depend on the GB crystallography. The latter result is particularly important when grain growth is investigated, since during grain growth migrating boundaries replace the shrinking ones and the crystallography of GBs continuously changes. This effect has not been taken into account during the above studies of grain growth under high 
pressure. The purpose of the present study is to investigate the effect of pressure on grain growth in aluminium using the recently developed experimental methods that permit to analyse the fraction of special and low energy GBs in the polycrystal during grain growth.

\section{EXPERIMENTAL}

\section{Material Preparation}

The experiments were carried out using foils of thickness in the range $0.08-0.1 \mathrm{~mm}$. The 2-D (columnar) structure was obtained by rolling of $99.999 \%$ purity aluminium to $90-95 \%$ reduction in thickness and subsequent annealing at $833 \mathrm{~K}$ for $20 \mathrm{~min}$. After annealing, a structure containing grains grown through the foil was obtained. The grain size was larger than the thickness of the foil and the grain boundaries were perpendicular to its surface. The mean grain area was $0.025 \mathrm{~mm}^{2}$. To investigate the pressure effect on grain growth, the foils were annealed at $773 \mathrm{~K}$ for $0.5,1,1.5,3,4$ and $6 \mathrm{~h}$ under atmospheric pressure and under the pressure of $1.2 \mathrm{GPa}$.

\section{The High Pressure Experiments}

The high pressure annealing was carried out under $99.999 \mathrm{wt} . \%$ argon atmosphere. The pressure was controlled by a managing gauge with the accuracy of $2 \%$. The temperature was controlled using three $\mathrm{Pt}$ $6 \mathrm{wt} . \% \mathrm{Rh}-\mathrm{Pt} 30 \mathrm{wt} . \% \mathrm{Rh}$ thermocouples with an accuracy of $1 \mathrm{~K}$. A three-zone high pressure furnace permitted to maintain a constant temperature over a plateau of $40 \mathrm{~mm}$ length, so that the specimens were in a uniform temperature field. The high accuracy of the temperature control was crucial to obtain meaningful results. According to Molodov et al. (1994) the displacement $l$ of a GB during annealing under high pressure is

$$
l=v t=t v_{0} \exp (-Q / R T) \exp \left(-p V^{*} / R T\right),
$$

where $v$ is rate of GB motion, $t$ is the annealing time, $v_{0}$ is the preexponential factor. If $\delta T$ is the range of temperature fluctuations, 
$\delta p$ is the range of pressures applied, and $\delta t$ is the accuracy of time measurement, the contribution of different constituents at constant temperature to the error in GB displacement is

$$
\Delta l / l=\left(Q / R T^{2}\right) \delta T+\left(V^{*} / R T\right) \delta p+\delta t / t .
$$

If $Q \sim 10 \mathrm{~kJ} / \mathrm{mol}, T \sim 10^{3} \mathrm{~K}, V^{*} \sim 10 \mathrm{~cm}^{3} \mathrm{~mol}^{-1}$ and $\delta T \sim 1 \mathrm{~K}$, the influence of the fluctuations in temperature on the GB displacement is in the order of $15 \%$ of the pressure effect for a pressure on the order $0.1 \mathrm{GPa}$. It is impossible to hold the temperature constant inside the high pressure cell with a higher accuracy than $1 \mathrm{~K}$. Therefore, to obtain meaningful results for the pressure effect, the pressure applied must exceed $1 \mathrm{GPa}$. These conditions were fulfilled in the present experiments. It has to be pointed out that in the present experiment thin aluminium foils were annealed under pressure. This is possible without deformation only under high gas pressure.

\section{Structure Investigation Techniques}

The mean grain size for the initial state of the specimens was measured using optical metallography. GBs etch to a different degree depending on their energy. During metallographic investigations in the optical microscope in bright field conditions the low energy GBs produce a weak contrast and it is practically impossible to reveal them. However, we performed an etching and observation procedure that permitted to reveal the low energy GBs using dark field conditions, an inclined beam and a large aperture. The angle of incidence of the light beam on the specimen surface was varied by rotating the microscope stage. This caused the variation in intensity of the grain colouring and permitted to obtain data on the grain size distribution in the foil including the low energy GBs. The technique for grain size determination that we used is described in the paper of Stoyan et al. (1987).

The obtained data allowed to determine mean grain size as well as to trace the evolution of the topological characteristics of the grain structure in the foils. (The topological class of the grain is the number of triple junctions belong to this grain.) One of the most important indications of the normal grain growth is the linearity of mean grain 
area vs. annealing time (Fradkov and Udler, 1994). An additional confirmation of normal grain growth is the time independent topological class distribution and linearity of the mean topological class vs. relative grain area.

Determination of grain misorientations was performed using the Scanning Electron Microscope and the Electron Channelling Pattern (ECP) technique (Sursaeva and Shvindlerman, 1995).

\section{RESULTS}

Figure 1 shows the time dependence of the mean grain area for annealing at atmospheric pressure and under the pressure of $1.2 \mathrm{GPa}$. It is seen that the grain growth rate decreases under high pressure by a factor 1.3. According to Eq. (2) the value for the activation volume, $V^{*}=1.310^{-6} \mathrm{~m}^{3} \mathrm{~mol}^{-1}$ or $V^{*}=0.13 \Omega$ was obtained.

Figure 2 shows the topological class distribution as a function of time. It is seen that the topological class distribution is time independent

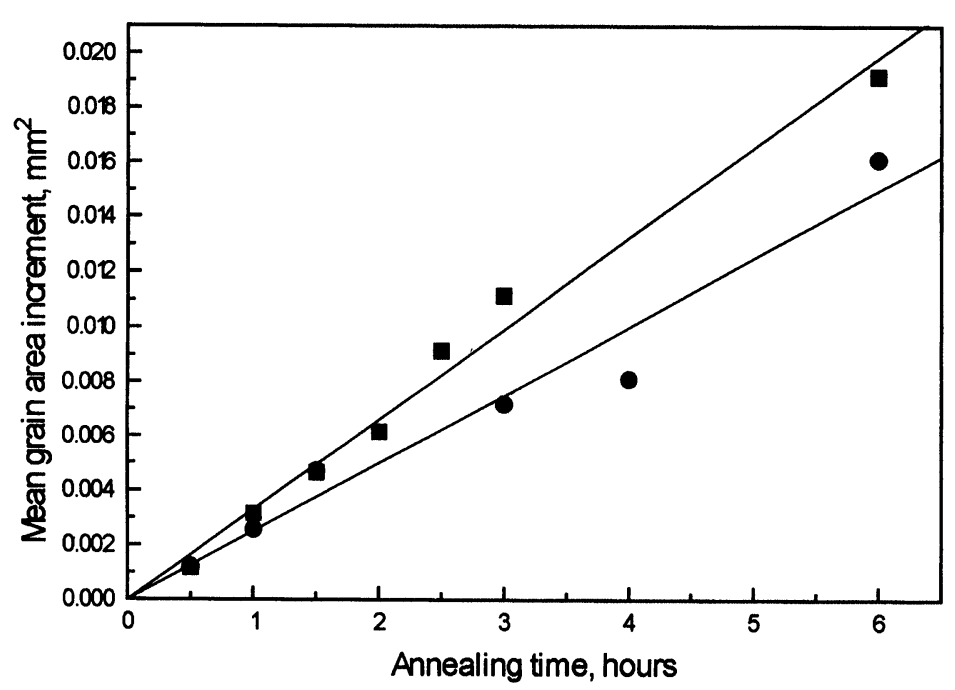

FIGURE 1 Time dependence of mean grain area increment at $T=773 \mathrm{~K}(\bullet)$ at the hydrostatic pressure $1.2 \mathrm{Gpa}$; $(\square)$ at the atmospheric pressure. 


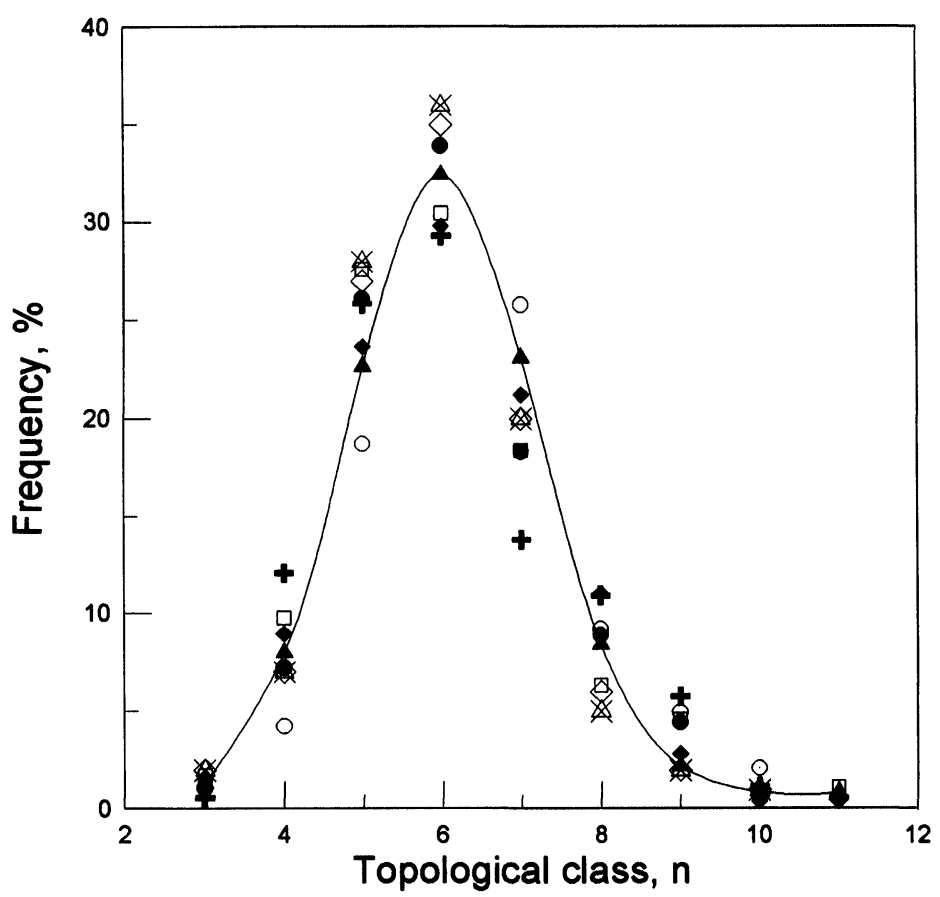

FIGURE 2 Topological class distribution. (০) $t=0.5 \mathrm{~h}$; (•) $t=1 \mathrm{~h} ;(\Delta) t=1.5 \mathrm{~h}$; (њ) $t=3 \mathrm{~h} ;(\square) t=4 \mathrm{~h} ;(\diamond) t=6 \mathrm{~h}$ at the hydrostatic pressure $1.2 \mathrm{Gpa} ;(\Delta) t=1.5 \mathrm{~h}$; (×) $t=3 \mathrm{~h} ;(\diamond) t=6 \mathrm{~h}$ at the atmospheric pressure.

as expected for normal grain growth (Fradkov and Udler, 1994; Shvindlerman et al., 1994a,b,c).

Figure 3 shows the linear relationship between mean topological class and the relative grain area.

Figure 4 shows the time dependence of the fraction of low energy GBs in the specimen. Up to $4 \mathrm{~h}$ of annealing time there is no difference in the fraction of low energy GBs for specimens annealed under high and low pressure. The fraction of low energy GBs increases up to about $50 \%$ of the total number of GBs. However, upon further annealing, the behaviour of the specimens annealed under high and low pressure is different. For the specimens annealed at atmospheric pressure the fraction of low energy GBs decreases to the level of about $22 \%$ (Sursaeva et al., 1997) while it slightly increases above the $50 \%$ level for the specimens annealed at the pressure of $1.2 \mathrm{GPa}$. 


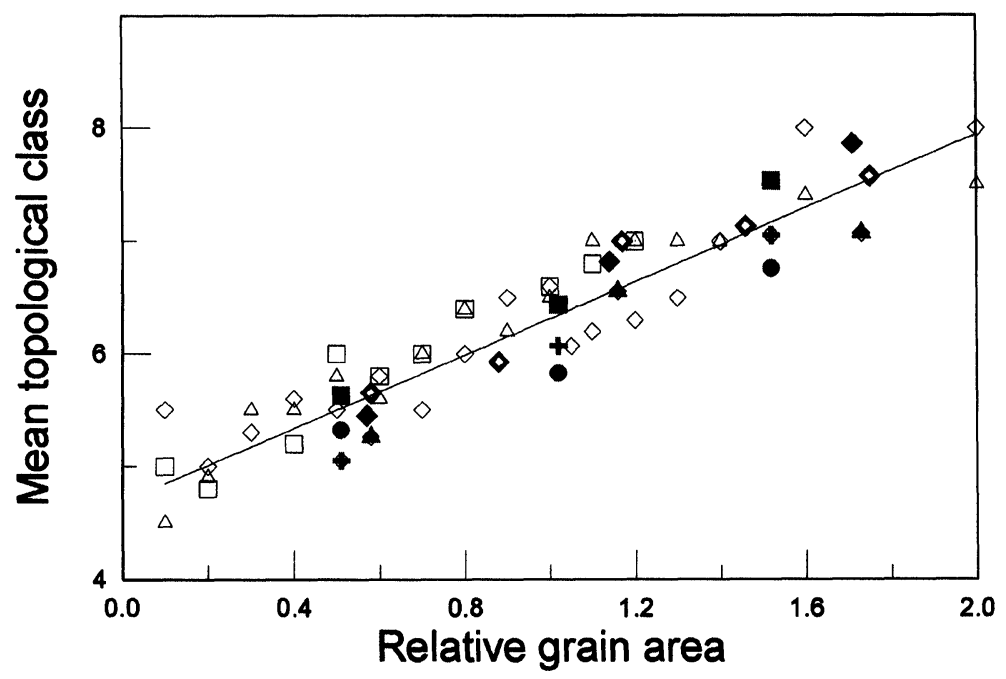

FIGURE 3 The mean topological class $\bar{n}(\mathbf{S})$ of the grains versus relative area $S / \bar{S}$.

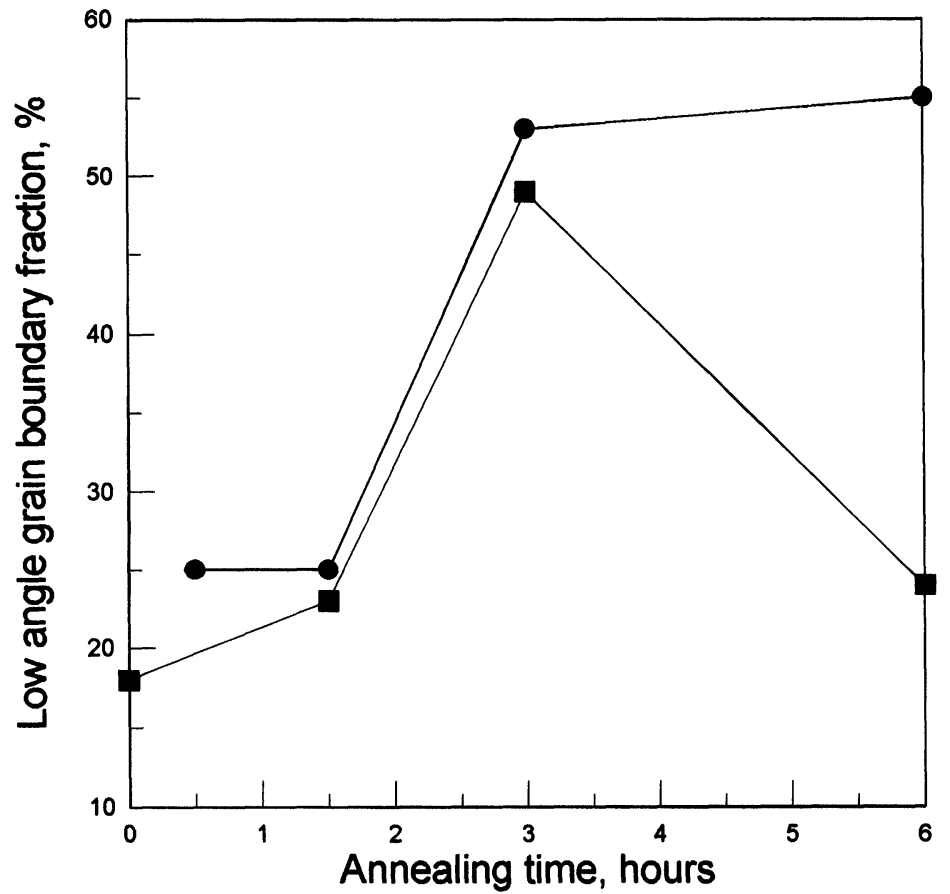

FIGURE 4 Time dependence of low angle grain boundary fraction at $T=773 \mathrm{~K}$ $(\bullet)$ at the hydrostatic pressure $1.2 \mathrm{Gpa}$; (๘) at the atmospheric pressure. 


\section{DISCUSSION}

So far all the papers known to us concerning grain growth have been dealing with three dimensional (3-D) specimens (Knyazev and Titorov, 1985; Rybin et al., 1984). X-ray methods were used to obtain statistical information about the distribution of GB misorientations. The data obtained in this way provide some averaged information. In contrast, the study of 2-D polycrystals permits to obtain the crystallographic data of each grain in the sample and permits a more straightforward identification of the mechanisms of grain growth (Shvindlerman et al., 1994a,b,c).

It is known that the process of recrystallization includes three different stages: primary recrystallization, normal grain growth and secondary recrystallization.

The changes of the mean grain area vs. annealing time are governed by a specific law at each stage. At the stage of normal grain growth there is a linear mean grain area dependence on time (Fradkov and Udler, 1994).

The linear grain growth rate (Fig. 1) and constancy of the grains mean topological class vs. relative area (Fig. 3 ) indicates that in our samples the primary recrystallization was accomplished before the high pressure annealing. In that respect, the grain growth process during the present experiments takes place in the same way as in the previous papers concerning this system (Shvindlerman et al., 1994a,b,c). Application of high pressure in the present study of grain growth permitted to obtain two new results: the activation volume for normal grain growth in 2-D aluminium foils and the pressure effect on the fraction of lowenergy GBs. As far as the activation volume is concerned, the value obtained, $V^{*}=0.13 \Omega$ is significantly less than that obtained for migration of individual GBs in aluminium bicrystals (Molodov et al., 1994). The $V^{*}$ values obtained by Molodov et al. were in the range $1-3 \Omega$, the exact value depending on the GB crystallography. The GBs studied were of symmetrical tilt type, with tilt axis $\langle 001\rangle,\langle 111\rangle$ and $\langle 011\rangle$. The highest values of the activation volumes were obtained for the $\langle 011\rangle$ tilt GBs. The above results were explained drawing attention to the fact that the $\langle 011\rangle$ tilt GBs have the most tightly packed structure.

On the other hand, the present value of the activation volume matches in part with the results of Lojkowski et al. (1995) obtained for grain 
growth in $\mathrm{Al} 4 \mathrm{~N}$. Lojkowski et al. (1995) studied the effect of temperature and material purity on the activation volume for grain growth in polycrystalline aluminium. They have found that the activation volume for grain growth was $0.2 \Omega$ for $\mathrm{Al} 5 \mathrm{~N}$ annealed at $560 \mathrm{~K}$. The same value was found for grain growth in $\mathrm{Al} 4 \mathrm{~N}$ at the temperature above $700 \mathrm{~K}$. On the other hand, the activation volume for grain growth at temperatures below $580 \mathrm{~K}$ was $0.7 \Omega$. Lojkowski et al. (1995) concluded that the high activation volume for grain growth in $\mathrm{Al} 4 \mathrm{~N}$ at low temperatures is caused by interaction of GBs with impurities. They postulated that at high temperatures impurities desegregate from GBs and they take an open structure permitting a vacancy-less migration mechanism. Gleiter and Lissowski (1971) proposed such a mechanism, where GB migrates owing to movement of the contacts between atoms, involving very small movements of atoms. This mechanism can be called "atomic bonds migration mechanism" for GB migration. On the other hand, they postulated that for the GBs with segregated impurities transfer of atoms perpendicular to the GB takes place with the cooperation of vacancies. It seems that this mechanism is active at low temperatures, where impurities segregate to GBs. Interaction with segregated impurities could also explain the high activation volumes found by Molodov et al. (1994).

When comparing GB migration in polycrystals and bicrystals, one has to take into account that the coverage of the GB by impurities is much higher in the latter case, since for a constant number of impurity atoms in a bicrystal there is only one GB. Furthermore, this GB sweeps all the volume of the bicrystal and is able to approach all the impurity atoms. Another possibility to explain the discrepancy of activation volumes measured for bicrystals and polycrystals is that in the case of polycrystals GBs with arbitrary axes and less close packed structures dominated the average grain growth rate, while the less mobile GBs with simple misorientation axis could not contribute to the average activation volume. In conclusion, it seems that the prevailing GB migration mechanism in polycrystals is the bond migration one. The vacancy mechanism becomes active presumably in GBs with densely packed structure that may result from impurity segregation or from good atomic matching of the adjoining crystals.

Let us discuss in detail the observed effect of pressure on the fraction of low energy GBs (Fig. 4). 
A strong texture in the 2-D foils with a sharp $\left\{\begin{array}{lll}0 & 0 & 1\end{array}\right\}\left\langle\begin{array}{lll}0 & 0 & 0\end{array}\right.$ component was revealed by Scanning Electron Microscopy and the ECP technique. It was shown that the 2-D polycrystal is composed of two regions. In the region of the first type the grains are oriented in such a way that the $\langle 100\rangle$ axis is normal to the surface of the foil. The GBs are mostly low angle, but some special ones are present. This region was called the $\langle 100\rangle$ colony. At the early stage of grain growth the $\langle 100\rangle$ colonies occupied $60-70 \%$ of the foil's area. The grains in the region of a second type had an arbitrary axis normal to a surface. They have been called general colonies. The boundaries between the grains were of high-angle type and the mobility of these GBs was higher than that of the low-angle ones. The general colonies occupied about $30-40 \%$ of the foil's area.

During grain growth the size of the grains within the $\langle 100\rangle$ colonies changed insignificantly because the mobility of the low-angle boundaries was low. However, the area occupied by these colonies expanded due to the movement of the high-angle boundaries surrounding the colony. It resulted in a decrease of the share of randomly oriented grains, whereas the number of the grains with the $\langle 100\rangle$ orientation and of low-angle GBs increased.

Taking into account the above results it seems that the pressure effect on grain growth becomes significant when the $\langle 100\rangle$ colonies absorbed major part of material. At that point further grain growth is controlled by the mobility of low-energy GBs. It is plausible that low-energy GBs are well ordered and can migrate only by the vacancy GB migration. However, the vacancy concentration in the material decreases with increasing pressure and the migration of low-energy GBs is slowed down stronger than in the case of general ones. As a consequence, the number of low-energy GBs is constant, as seen in Fig. 4. The change of low-angle GBs fraction due to a pressure effect on texture seems unlikely taking into account the stability of the texture in the specimens investigated.

\section{CONCLUSIONS}

1. It was observed that the fraction of low-angle grain boundaries during normal grain growth under hydrostatic pressure is considerably higher compared to atmospheric pressure. Authors 
associate this effect with a stronger decrease of the low-angle boundary mobility than that of the high-angle ones.

2. The average activation volume for grain growth was 0.13 of the atomic volume and is compatible with grain boundary migration by movement of inter-atomic bonds.

\section{Acknowledgements}

The authors are grateful to Prof. Shvindlerman for fruitful discussions. The financial support of the Russian Foundation for Fundamental Research (grant RFFI 95-02-05487a), Polish Committee for Scientific Research and the University of Ulm is acknowledged.

\section{References}

Buescher, B.J., Gilder, H.M. and Shea, N. (1973). Phys. Rev. B, 7, 2262.

Curtin, H.R., Decker, D.L. and Vanfleet, H.B. (1965). Phys. Rev. A, 139, 1552.

Erdelyi, G., Lojkowski, W., Beke, D.L., Godeny, I. and Kedves, F.J. (1987). Phil. Mag. A, 56, 637.

Fradkov, V.E. and Udler, D. (1994). Advances in Physics, 43, 739.

Gleiter, H. and Lissowski, A. (1971). Zeitschrift fur Metallkunde 62, 237.

Hahn, H. and Gleiter, H. (1979). Scripta Metall., 14, 3-6.

Kedves, F.J. and Erdelyi, G. (1989). Defect and Diffusion Forum, 66-69, 57.

Knyazev, N.M. and Titorov, D.B. (1985). Fiz. Metal. Metallov., 59, 780.

Kohler, U., Mehrer, H. and Lojkowski, W. (1992). Phys. Stat. Sol. (b), 174, 347.

Lojkowski, W. (1996). Defect and Diffusion Forum, 129-130, 269.

Lojkowski, W., Minamino, Y., Hozumi, S. and Wyrzykowski, J. (1995). Proc. Joint XV AIRAPT\& XXXIII EHPRG Intern. Conf. on High Pressure Science and Technology, Warsaw, Poland, September 11-15.

Martin, C., Blackburn, D.A. and Adda, Y. (1967). Phys. Stat. Sol. (a), 23, 223.

Mehrer, H. (1996). Defect and Diffusion Forum, 129-130, 57.

Molodov, D.A., Straumal, B.B. and Shvindlerman, L.S. (1984). Sov. Phys. Solid State, 26, 629.

Molodov, D.A., Swiderski, J., Gottstein, G., Lojkowski, W. and Shvindlerman, L.S. (1994). Acta Metall. Mater., 42, 3397.

Rybin, V.V., Titovetz, Yu.F. and Kozlov, A.L. (1984). Poverkhnost, 10, 107.

Shvindlerman, L.S., Sursaeva, V.G., Novikov, V.Yu. and Faulkner, R.G. (1994a). Materials Science Forum, 157-162, 1063.

Shvindlerman, L.S., Sursaeva, V.G., Yashnikov, V.P. and Faulkner, R.G. (1994b). Interface Science, 2, 153-168.

Shvindlerman, L.S., Sursaeva, V.G., Yashnikov, V.P. and Faulkner, R.G. (1994c). Materials Science Forum, 157-162, 1057.

Stoyan, D., Kendall, W.S. and Mecke, J. (1987). "Stochastic Geometry and its Applications". Berlin: Akademie-Verlag, 276.

Sursaeva, V.G. and Shvindlerman, L.S. (1995). Proc. 16th RisØ Int. Symp. On Materials Science, Roskilde, Denmark, pp. 559-564.

Sursaeva, V.G. et al. (1997). "Grain and Grain Boundary Structure Evolution without Textures Changes During Normal Grain Growth in 2-D AlStrips". These proceedings.

Vieregge, K., Herzig, Ch. and Lojkowski, W. (1991). Scripta Metall. Mater. 25, 1707. 